\title{
Pesticides and your food: How safe is "safe"?
}

\author{
Gary A. Beall \\ Christine M. Bruhn \\ Arthur L. Craigmill \\ Carl K. Winter
}

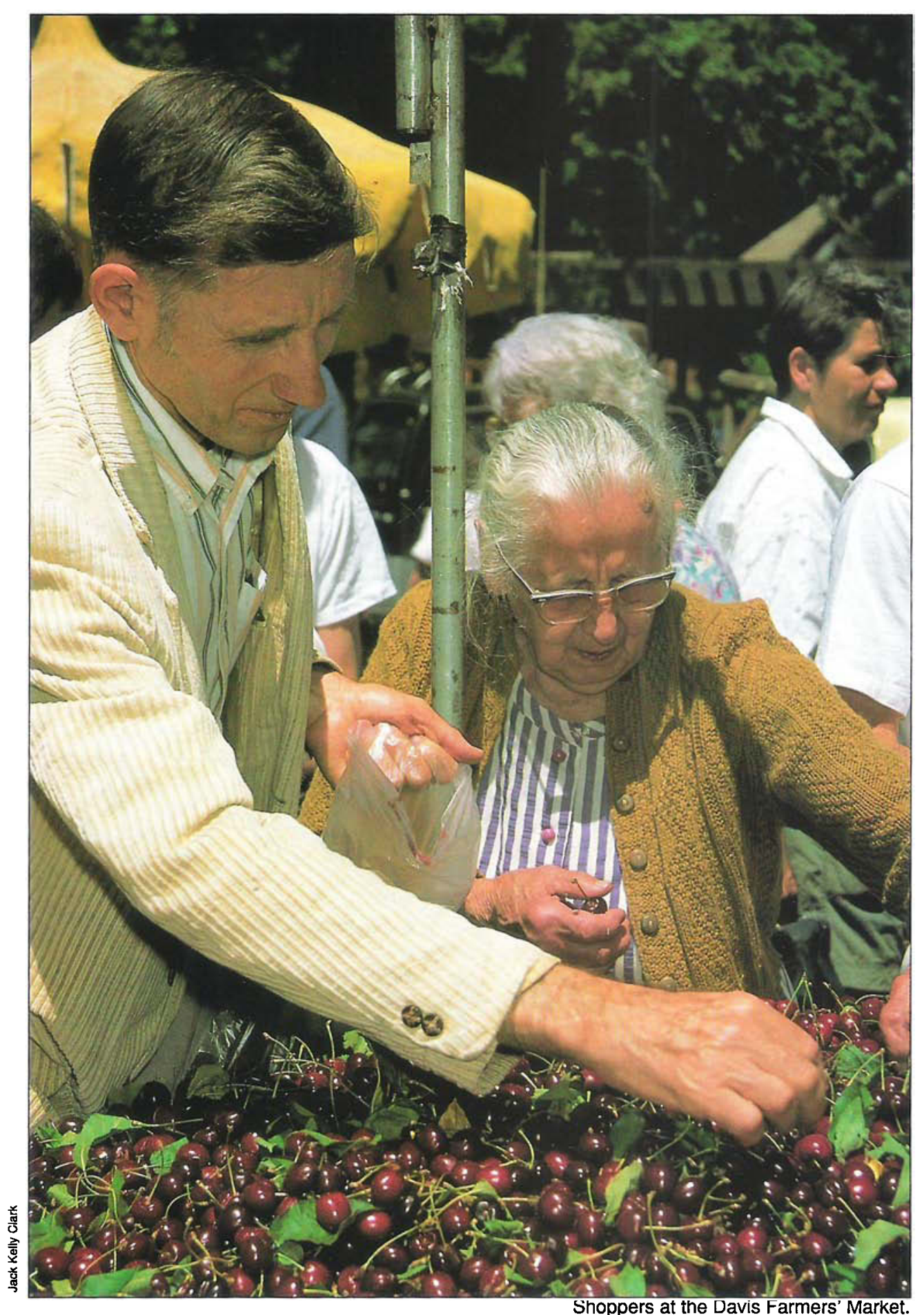

Shoppers at the Davis Farmers' Market.
While public confidence in the fresh food supply has rebounded in recent months, consumers still identify pesticide residues as a major food safety concern - and $8 \%$ of Californians say they buy less fresh produce as a result.

This paper explains how pesticides in food are regulated, explores the effectiveness of these regulations, and offers alternatives to consumers who are not satisfied with the current process. It was prepared by four members of a diverse, $U C$-sponsored committee which met for 20 months to examine the university's food safety research and education.

Appointed by Kenneth Farrell, Vice President of UC's Division of Agriculture and Natural Resources, committee members came from consumer and environmental groups, the California Legislature, farm and food industries, the public health community, the University of California, the California Department of Food and Agriculture, and Stanford University.

Although the diverse membership did not reach consensus on all issues, members clarified their own understandings about food safety. The following article will be used by UC as an educational tool throughout the state.

$\mathrm{I}_{\mathrm{n}}$ In February, 1989, "60 Minutes" aired the Natural Resources Defense Council findings that Alar, a chemical growth regulator used on some apples, posed potential health risks for school children. Food safety subsequently made the cover of Time, and was treated by Newsweek and Phil Donahue, among others. Apples and apple juice disappeared from many school cafeterias.

In March of the same year, two grapes imported from Chile were found to be 


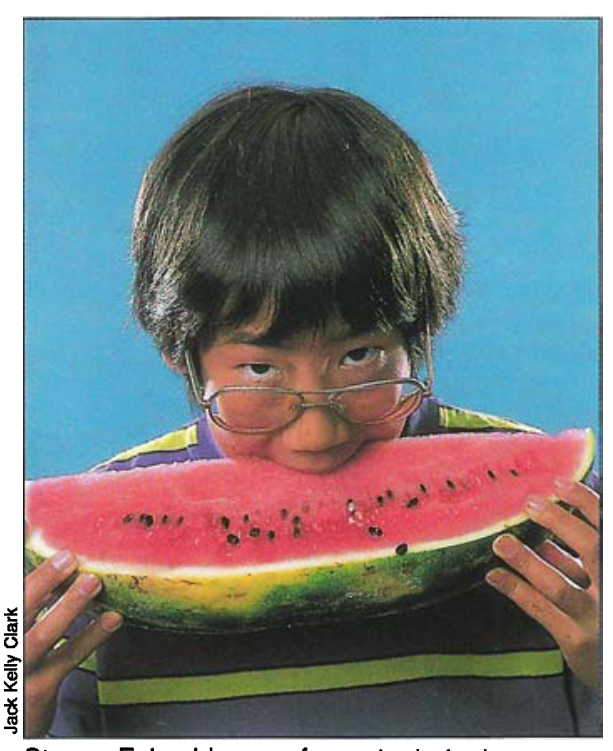

Steven Fukushima performs taste test.

contaminated with cyanide (an apparent case of tampering). All Chilean fruit already in the U.S. at the time was then withdrawn from the market.

The profound psychological impact of these events on supermarket shoppers is now well documented. In January of 1989 $81 \%$ of consumers polled said they were completely or mostly confident that the food in their supermarket was safe. That figure plummeted to $67 \%$ two months later, according to surveys conducted by the Food Marketing Institute.

The Packer Focus Fresh Trends '90 survey revealed that consumer confidence was slow to rebuild. That survey reported that $17 \%$ of shoppers nationwide were buying less fresh produce because of concerns about pesticide residues. In a more recent UC survey of California shoppers, $8 \%$ volunteered they had curtailed some purchases of fresh produce.

Although the National Research Council (of the National Academy of Sciences) has concluded that fruits and vegetables help reduce the likelihood of cancer, consumers are not convinced. The Packer survey found that only $54 \%$ agreed that "the potential health benefits of eating fresh fruits and vegetables outweigh the potential risks from pesticide residues." Almost half $-46 \%$ - disagreed.

Because consumers are faced with increasingly complex and often contradictory information about food safety, the following report is intended to provide them with a basic account of current regulatory practice, and a context in which to understand the current controversies.

\section{What is acceptable risk?}

Both the Environmental Protection Agency (EPA) and the Food and Drug Administration (FDA) say that diet is the most significant long-term source of pesticide exposure for the general public. But authorities disagree on how much risk is posed by pesticides. They also differ on what is "acceptable risk."

In some instances, the general public, policy makers or consumer and environmental advocates support a "zero risk" concept. An example is the 1958 Delaney Amendment to the Food, Drug and Cosmetic Act. The amendment states that no food additive should be allowed if it is a known carcinogen. Another piece of legislation which defines risk is California's Proposition 65, stating that significant risk is one hypothetical cancer-related case in 100,000 . In general, however, laws regulating pesticides do not define acceptable and unacceptable risk.

While the Food and Drug Administration (FDA) implements some parts of the Food, Drug and Cosmetic Act, the Environmental Protection Agency (EPA) implements the sections concerning pesticides. Several congressional acts empower the EPA to regulate pesticides - whether in food, the environment or toxic waste. Regulation stems from standards set during pesticide registration. By law, each registration is based upon a risk-benefit analysis.

It is this analysis of benefits and risks that creates much of the controversy surrounding the use of pesticides. Benefits and risks are not perceived the same way by everyone and they seldom are equally distributed.

Benefits may translate into longer cropgrowing seasons, larger yields, and more attractive, affordable food products that have less waste and a longer shelf life. Risks may translate into pesticide applicator and farmworker hazards; adverse ef-

Figs. 1 and 2. Government agencies use different methods to regulate cancer-causing and noncancer-causing substances.

Figure 1 shows how safety factors are used to set safe exposure levels for noncarcinogens. These safety factors are applied to data from animal studies in the laboratory. The starting point is the No Observable Effect Level (NOEL) which is the highest dose which did not produce an effect. By dividing the NOEL by an appropriate safety factor, the Acceptable Daily Intake (ADI) is established.

Figure 2 shows how exposure levels for carcinogenic effects are established from laboratory data. Regulators use the risk assessment model developed from data on the most sensitive animal species tested. The lowest dose that produced a measurable response in animals is used as the starting point. A line is drawn from this point to the origin (0) on the response axis. The scale is expanded, and the dose which is predicted to pose negligible risk (one additional cancer in 1 million people) is chosen as the regulatory limit. fects on the environment such as ground water contamination, and consumer exposure to residues on the food they eat.

\section{Zero risk vs. negligible risk}

The scientific community generally agrees that the concept of "zero risk" associated with human health is unrealistic because everything we do has some risk associated with it. For example, if the government were to ban all fungicides that present a cancer risk, fungi in fresh produce would increase. Fungi manufacture their own natural carcinogens (such as aflatoxins) in significant amounts.

In assessing risk, scientists and regulators use different procedures for carcinogens and noncarcinogens. (See figs. 1 and 2.) The concept of "negligible risk" or "no significant risk" is widely accepted as the basis for carcinogen risk assessment studies. A cancer-causing substance is said to pose negligible risk if the population's exposure to this substance at its regulatory limit could potentially cause one additional cancer in a million people.

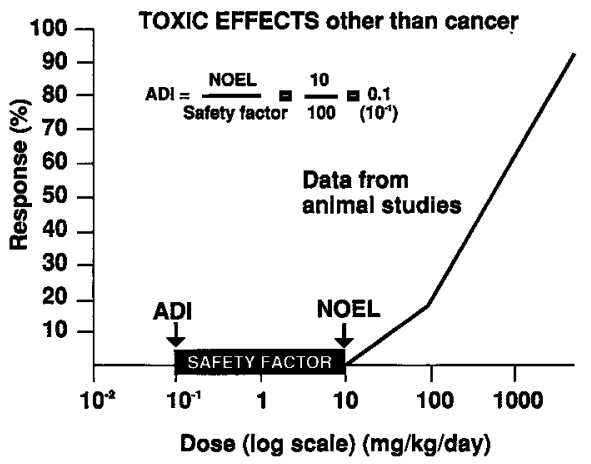

Fig. 1. Hypothetical calculation of ADI for a substance with potential toxic effects other than cancer.

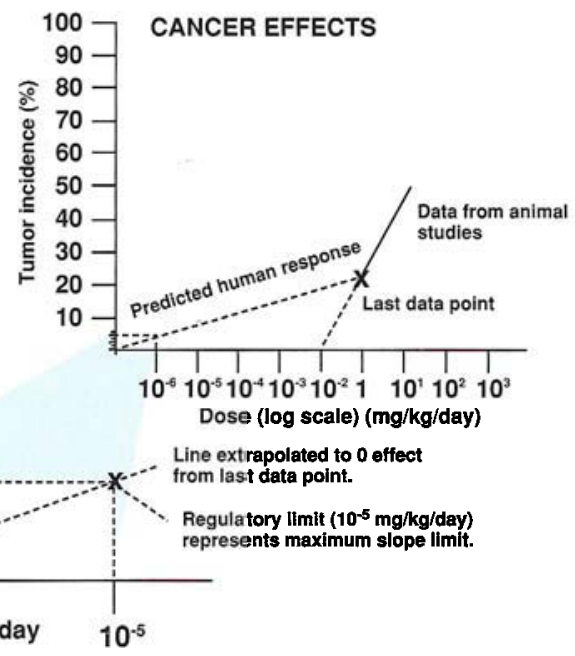

Fig. 2. Hypothetical calculation of a regulatory limit for a substance with cancer-causing effects. 


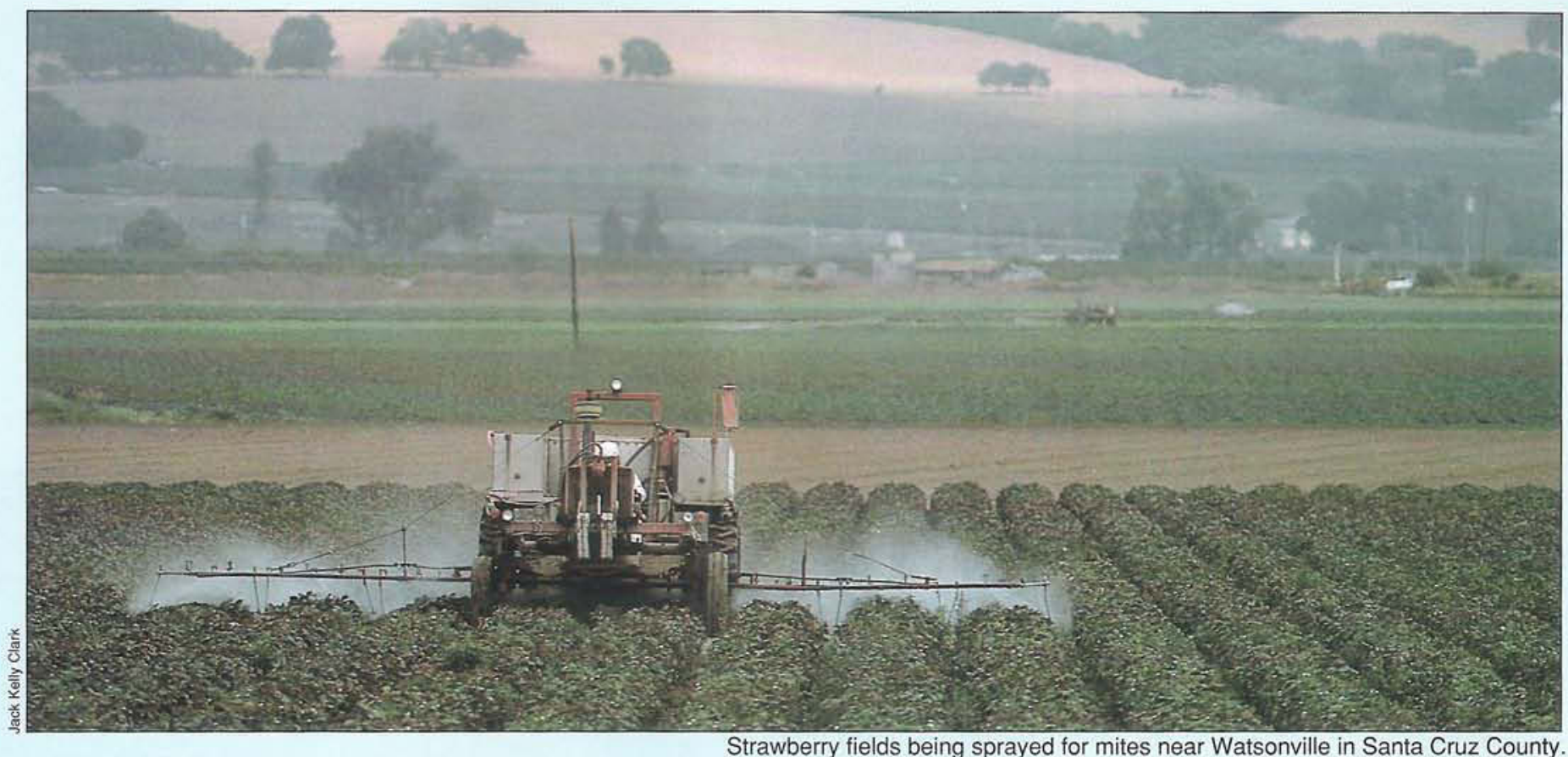

\section{Pesticides in food: major controversies}

The evidence we have suggests that pesticide residues on food pose only negligible risk in the American diet, but some evidence is unavailable. Scientific uncertainties have given rise to several controversies. Other debates concern practical difficulties in pesticide regulation.

\section{Reregistration: the EPA backlog}

The great majority of pesticides in use today were registered before extensive toxicology testing was required. This does not mean that no studies were done, but rather that older studies no longer meet current standards. Legislation in 1972 called for re-registration of these pesticides. However, of 822 active ingredients in current use, only two have been re-registered to date. Of the same 822 pesticides, about 350 receive uses which may result in residues on food.

Congress in 1988 accelerated the timetable for a national re-registration, requiring pesticide manufacturers to fill in "data gaps" by 1997 to determine whether these older products should maintain their registrations (FIFRA, 1988). Critics of the system say the re-registration process is moving too slowly and certain older pesticides should not remain in use in the interim.

Possible reasons for re-registration delays are time and money. The cost of developing and registering a new pesticide has been estimated to exceed \$20 million. The time required to complete long-term animal studies - just one portion of required tests - is four to five years. Manufacturers may prefer not to make this investment in little used chemicals.

\section{Actual residues vs. tolerances}

National studies have sometimes used maximum "tolerance levels" to estimate actual pesticide residues on food. These tolerance values - set in the registration process - are many times higher than actual residue levels found in government monitoring programs.

Also, food processing, transportation and handling practices (such as washing, peeling, and cooking) can dramatically decrease the amount of residue that is ultimately consumed. However, some food processes such as concentration or dehydration may magnify pesticide residues or breakdown products. A study of the combined effects of these processes found that pesticide residues in the final products were still below acceptable daily intake.

\section{Sample size in tests}

In all of these testing programs, less than $2 \%$ of our food supply is actually sampled. The Total Diet Study, for instance, includes four market baskets of food items, each from a different region of the country. It has been criticized for offering a low level of statistical significance.

Small sample size may also reduce the effectiveness of monitoring. Limited time, money and technology may allow illegal uses to go undetected. However, evidence indicates that most illegal residues are chemicals which have been used on crops for which they are not registered. They may have been wrongfully used or inadvertently picked up from neighboring crops or soil residuals. They are not necessarily present at harmful levels.
To compensate for small sampling size, monitoring programs are intentionally biased toward finding residues. Emphasis is placed on foods most likely to have residues and where problems have surfaced in the past. Regulators focus on the most used pesticides and those that pose the greatest health concerns.

\section{Inert ingredients}

Inert ingredients are not considered when setting tolerances. This means that there is limited health information on some of the 800 to 1,200 inert ingredients that are commonly used as solvents, stickers, spreaders and for other purposes in pesticide formulations. Inert ingredients may constitute a very large or very small portion of a formulation. The EPA has identified at least 50 of 1,200 possible inert ingredients which are of known toxicological concern.

\section{Interactive effects}

The effects of combinations of pesticides also are not usually considered. The most common way for chemicals to interact is to cause additive effects. That is, the combined effect of two or more chemicals equals the total of the individual effects. Chemicals may also be antagonistic, that is, the effects cancel each other. Or they may be synergistic, with the combined effect being more than the additive effect of the pesticides. Regulators believe that safety factors built into the risk management process compensate for these potential interactions, and for differences in sensitivity among people. 
Although it is not written into law, the concept of negligible risk is used in the risk-benefit analysis. Several conservative assumptions are normally applied. For instance, cancer risks are typically expressed as the "upper bound" or the highest incidence expected, based upon a theoretical model; the lower bound may be several orders of magnitude less and may, in fact, be zero. The regulatory limit is typically set at the $95 \%$ confidence limit, meaning that there is $95 \%$ probability that the health risk posed by that dose will not exceed one additional cancer in a million. people.

While risk estimates for potentially carcinogenic pesticides are useful for regulatory purposes, they do not predict actual human cancer incidence. EPA scientists use the worst-case scenario when predicting the effects of very low doses of pesticides on humans from the effects of very high doses on laboratory animals. Regulators may also make conservative assumptions about what is a possible carcinogen.

Negligible risk for noncarcinogens is also based upon conservative assumptions called "safety factors." Acceptable daily intake (ADI) values are usually calculated by reducing the maximum "no observable effect level" in experimental animals by a safety factor in the range of 10 to 1,000 (fig. 1). A 100-fold safety factor is common. This is based on the assumption that humans are 10 times more sensitive than the most sensitive animal species studied and that some humans are 10 times more sensitive than the least susceptible humans.

\section{The regulatory process}

Before a pesticide can be used on a crop it must be registered for use by the EPA. And before the EPA will consider registering a new pesticide, the company that developed the pesticide must submit the results of studies showing the pesticide's toxic effects on laboratory animals, at what doses it caused them, and studies of how the proposed pesticide behaves in the environment. (See box.) Studies showing the effects of the pesticide on non-target animals such as fish, birds and wildlife may also be required.

If the use of a pesticide has the potential to leave a residue on a particular food item, the EPA will require that pesticide residue studies be performed. These studies are done in a variety of geographical locations under the most severe application conditions - maximum application rate, maximum number of applications per year, and shortest interval between the last pesticide application and harvest.

At the conclusion of the residue studies, the maximum residue observed will be determined and the manufacturer of the pesticide will request that a tolerance, or maximum allowable residue level, be granted by the EPA that slightly exceeds the maximum residue observed.

The EPA then calculates the theoretical maximum (worst-case) consumer exposure to the pesticide from all foods. The EPA will usually not approve a tolerance unless total theoretical exposure is below the acceptable daily intake (ADI). The ADI is defined as the level of daily exposure to a pesticide residue which, over a 70-year human life span, will have no negative effect. "Reference dose," a new term in EPA parlance, is sometimes used for ADI.

ADIs are determined from toxicology studies using laboratory animals. Large doses of test chemicals are used to maximize the chances of finding any adverse health effects in a relatively short time span. The dose or exposure level that produces no noticeable toxic effect on test animals is reduced by a safety factor - usually 100-fold - and then is used as the basis for determining the ADI for humans. (See fig. 1)

For carcinogens, regulatory limits not ADIs - are calculated. Generally, the regulatory limit is the dose which poses negligible risk - that of causing one additional cancer in 1 million people. (See fig.

2.) This standard is commonly used in the risk-benefit analysis, but the EPA is not required to limit risk to a certain level. It is required to register pesticides based upon the entire picture of risks and benefits.

Inert ingredients are not considered when setting tolerances. This means that there is limited health information on some of the 800 to 1,200 inert ingredients that are commonly used as solvents, stickers, spreaders and for other purposes in the pesticide formulations. Inert ingredients may constitute a very large or very small portion of a formulation. The EPA has identified 50 of 1,200 possible inert ingredients which are of known toxicological concern.

The effects of combinations of pesticides also are not usually considered. The most common way for chemicals to interact is to cause additive effects. That is, the combined effect of two or more chemicals equals the total of the individual effects. Chemicals may also be antagonistic, that is, the effects cancel each other. Or they may be synergistic, with the combined effect being more than the additive effect of the pesticides. However, safety factors built into the risk assessment process and into setting the acceptable daily intake are presumed to compensate for potential interactions and for differences in sensitivity among people.

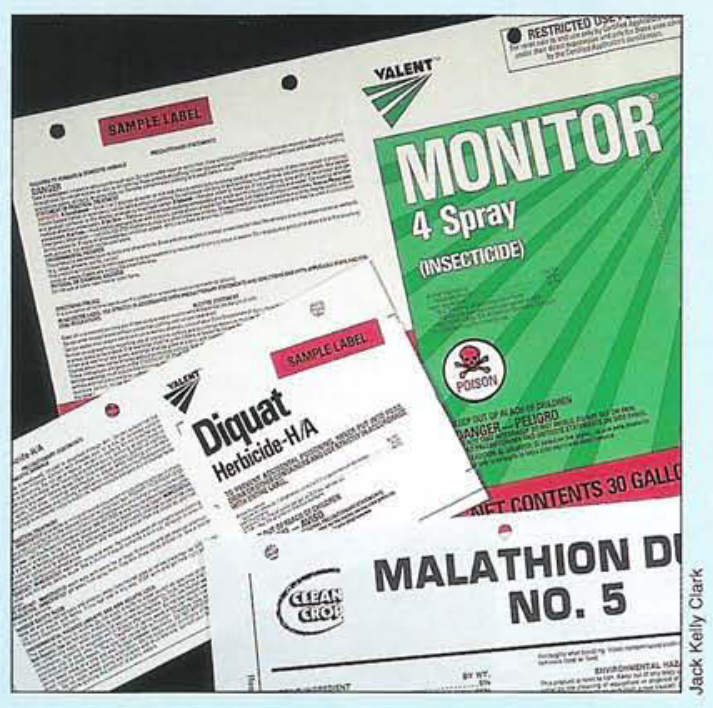

\section{EPA data requirements for pesticide registration}

\section{Acute toxicity}

oral toxicity - rat

dermal toxicity

inhalation toxicity - rat

primary eye irritation - rabbit

primary dermal irritation

dermal sensitization

acute delayed neurotoxicity - hen

\section{Subchronic toxicity}

90-day feeding studies - rodent and non-rodent

21-day dermal

90-day dermal

90-day inhalation - rat

90-day neurotoxicity - hen, mammal

\section{Chronic toxicity}

chronic feeding - 2 rodent species, one non-rodent

oncogenicity study (tumor-causing effects) -2 species (rat and mouse preferred)

teratogenicity (birth defects) - 2 studies

reproduction - 2 generations

\section{Mutagenicity testing}

gene mutation structural chromosomal aberration other genotoxic effects

\section{Special testing}

general metabolism dermal penetration domestic animal safety non-target species other

\section{Environmental fate}

residues on crops

ground water contamination 


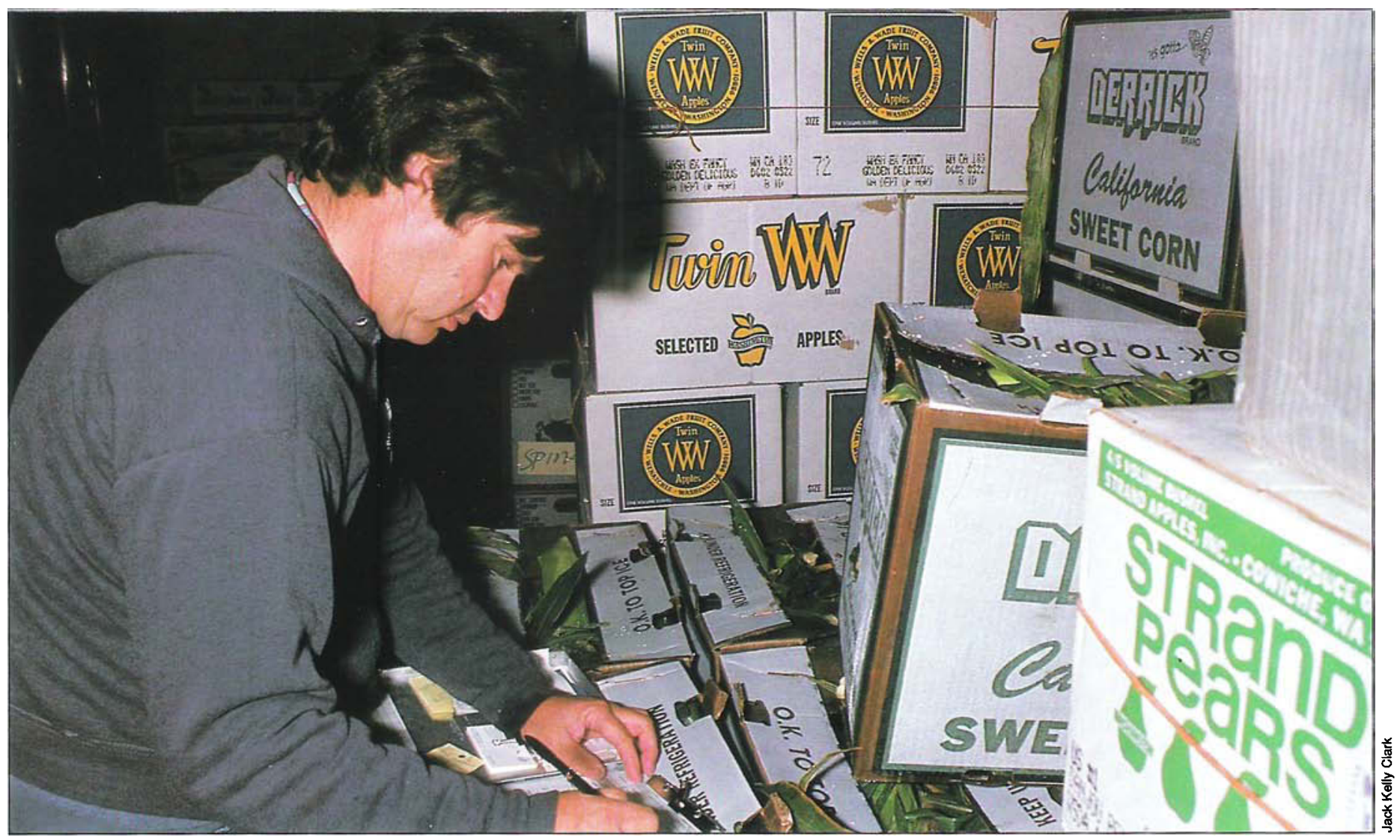

\section{What registration really means}

Registration of a pesticide means that it can be used to control specific pests on specific crops as long as certain conditions - such as application rate and timing are met. It also means that the EPA, after reviewing required research results, has concluded that the benefits from the use of the pesticide outweigh the risks.

These legal conditions for a pesticide's use must be on the label, along with additional information about protection needed for applying the product, minimum intervals between pesticide application and harvest, and guidelines on how to handle spills and other emergencies. It is illegal to apply a pesticide in a manner not specified on the label.

Although the ADI is a health-based standard, tolerances are enforcement standards. Residues that exceed tolerance values indicate pesticide misuse, but rarely create health risks because over-tolerance residues seldom cause consumer exposure to exceed the ADI. Even if the ADI is temporarily exceeded, such short-term exposure would rarely cause harm because the ADI is typically based on lifetime exposure.

Individual states have the right to require their own data in their own registration process. About half of the state governments, including California, have adopted even more stringent standards than the federal government in controlling the use of pesticides. California has the most comprehensive standards, requiring data relating to worker exposure, ground water contamination, medical management and other areas.

The great majority of pesticides in use today were registered before extensive toxicology testing was required. This does not mean that no studies were done, but rather that studies, adequate at the time, do not meet more comprehensive current standards. Legislation in 1972 called for reregistration of these pesticides. However, of 822 active ingredients in current use, only two have been re-registered to date. Of the 822 , about 350 are pesticides which may leave residues on food.

Congress in 1988 accelerated the timetable for national re-registration, requiring pesticide manufacturers to fill in "data gaps" by 1997 to determine whether these older products should maintain their registrations (FIFRA, 1988). Critics of the system say that the re-registration process is moving too slowly and that certain older pesticides should not remain in use in the interim.

\section{The monitoring and enforcement process}

Once established, tolerances are the basis for monitoring pesticide application practices. At the federal level, monitoring is done by the U.S. Food and Drug Administration (FDA). At the state level, it is done by the California Department of Food and Agriculture (CDFA). Independent laboratories also do testing on a contract basis with supermarkets and growers.

In 1989 , the FDA analyzed 18,113 food samples in their routine surveillance. Of
Tom Anderson, a pesticide use specialist for the CDFA, takes random samples at a commercial produce distribution center in West Sacramento.

these, $59 \%$ were from foreign countries. No residues were found in $66.3 \%$ of food samples tested; $31.2 \%$ contained legal, detectable residues, and $2.5 \%$ contained residues that exceeded tolerances or occurred on crops for which they were not registered.

The CDFA testing program in 1989 analyzed 14,987 samples of food in a variety of different programs. A majority of the samples - 9,403 samples from 179 different commodities - were taken from the "marketplace," which includes wholesale and retail markets, chain store distribution centers, packing houses, and points of entry into California. Of these samples, $54.8 \%$ were from food grown in California, $10.8 \%$ from food grown in other states, and $34.4 \%$ from foreign countries. No residues were detected in $78 \%$ of these marketplace samples. Residues within tolerance limits were found in $21 \%$. Illegal residues were detected in less than $1 \%$ of the samples, most of which were from pesticides not authorized for use on the commodity being tested. Fewer than one quarter of $1 \%$ of the samples showed residues above tolerance levels.

When monitoring food imports, the FDA and CDFA test for several pesticides which have been cancelled in the United States but which are still in use elsewhere. For example, the CDFA tests for DDT, 


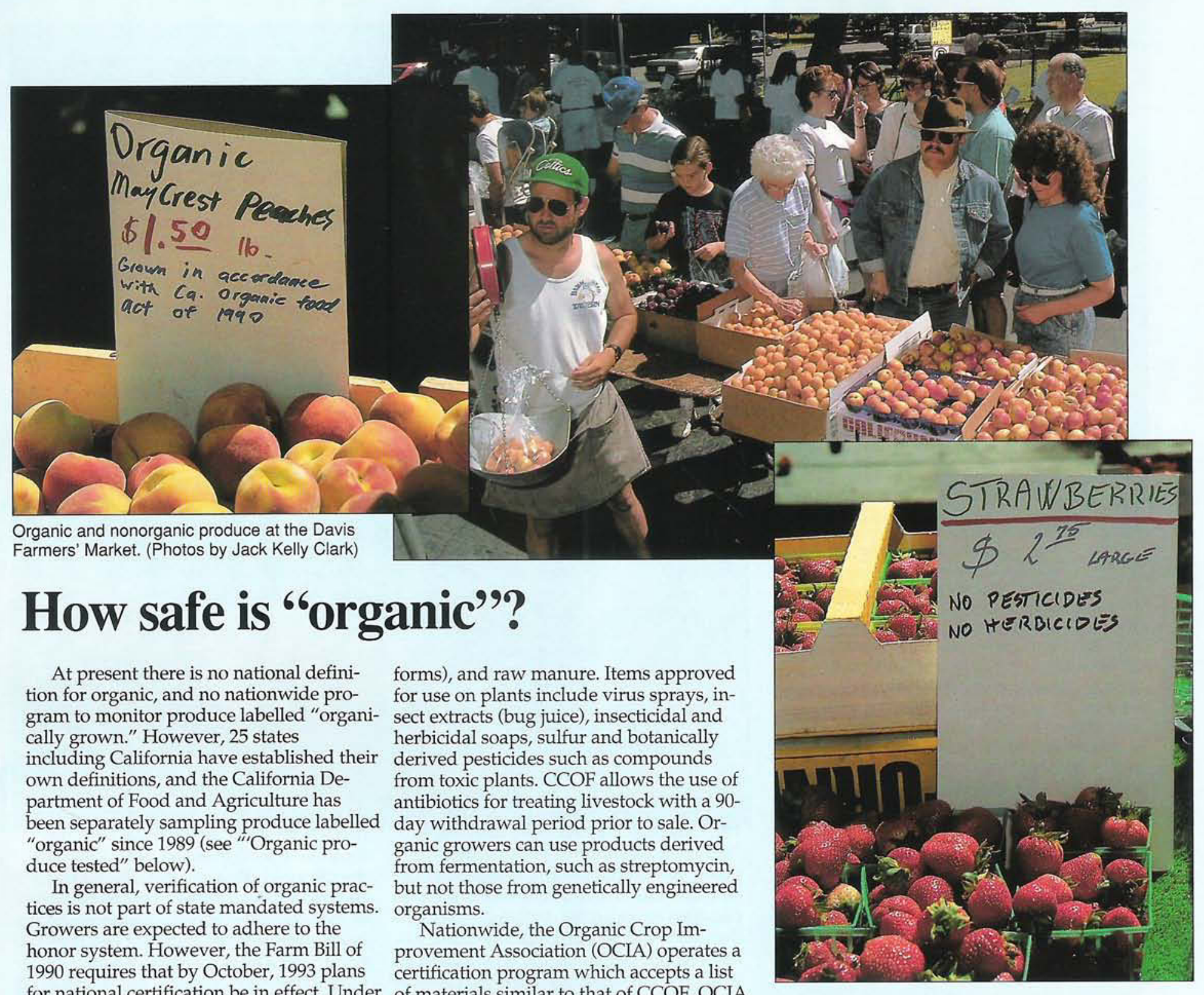
for national certification be in effect. Under the new federal system, states with organic certification will be able to continue their program if the system meets minimum requirements. A state may internally set more stringent requirements than the federal standard, but may not discriminate against imported products that meet the national standards.

\section{California guidelines}

In California, the California Certified Organic Farmers Association, CCOF, certifies its own members. CCOF guidelines draw a distinction between naturally occuring and synthetic compounds, and restrict use of synthetics. However, some synthetic materials are allowed under a "grandfather provision." Examples are copper sulfate, copper hydroxide and lime. These materials are presumed safe based on a history of use.

Natural materials approved for use by $\mathrm{CCOF}$ in soils include such items as bone meal, compost, gypsum (only mined

of materials similar to that of CCOF. OCIA evaluates chemicals on a case-by-case basis and prohibits most synthetic or refined ingredients.

Contrary to popular belief, organic farmers may use chemicals to balance risks and benefits. Naturally derived products are not without risks to the workerapplicator, the environment, and the consumer. A key component in safety is not the source of the chemical, be it natural or synthetic, but its toxicity and level of occurrence. In all cases, the dose makes the poison.

\section{Organic produce tested}

The California Department of Food and Agriculture began separately sampling food labelled as organically-grown in 1989. Of 198 samples tested that year, residues were detected in seven or 3.5\%. All were within tolerance. In 1990, tests of 197 samples revealed residues in four or $2 \%$, again all well within tolerance levels.

These results show that residue levels differ in organic and nonorganic produce, although residues found in nonorganic produce are still well within established margins of safety. In 1989, the FDA analyzed 18,113 food samples for pesticide residues during routine surveillance. Residues at very low levels (below tolerances) were found in $31.2 \%$ of the foods sampled; $2.5 \%$ contained residues that exceeded tolerance or occurred on crops for which they were not registered.

The same year the CDFA tested 9,403 samples of nonorganic produce taken from the "marketplace," including wholesale and retail markets, distribution centers, packing houses, and points of entry for foreign-grown produce. Residues within tolerance limits were found in $21 \%$. Illegal residues were found in less than $1 \%$ of the samples, most of which were from pesticides not authorized for use on the commodity being tested. Fewer than one quarter of $1 \%$ of the samples showed residues above tolerance levels. 


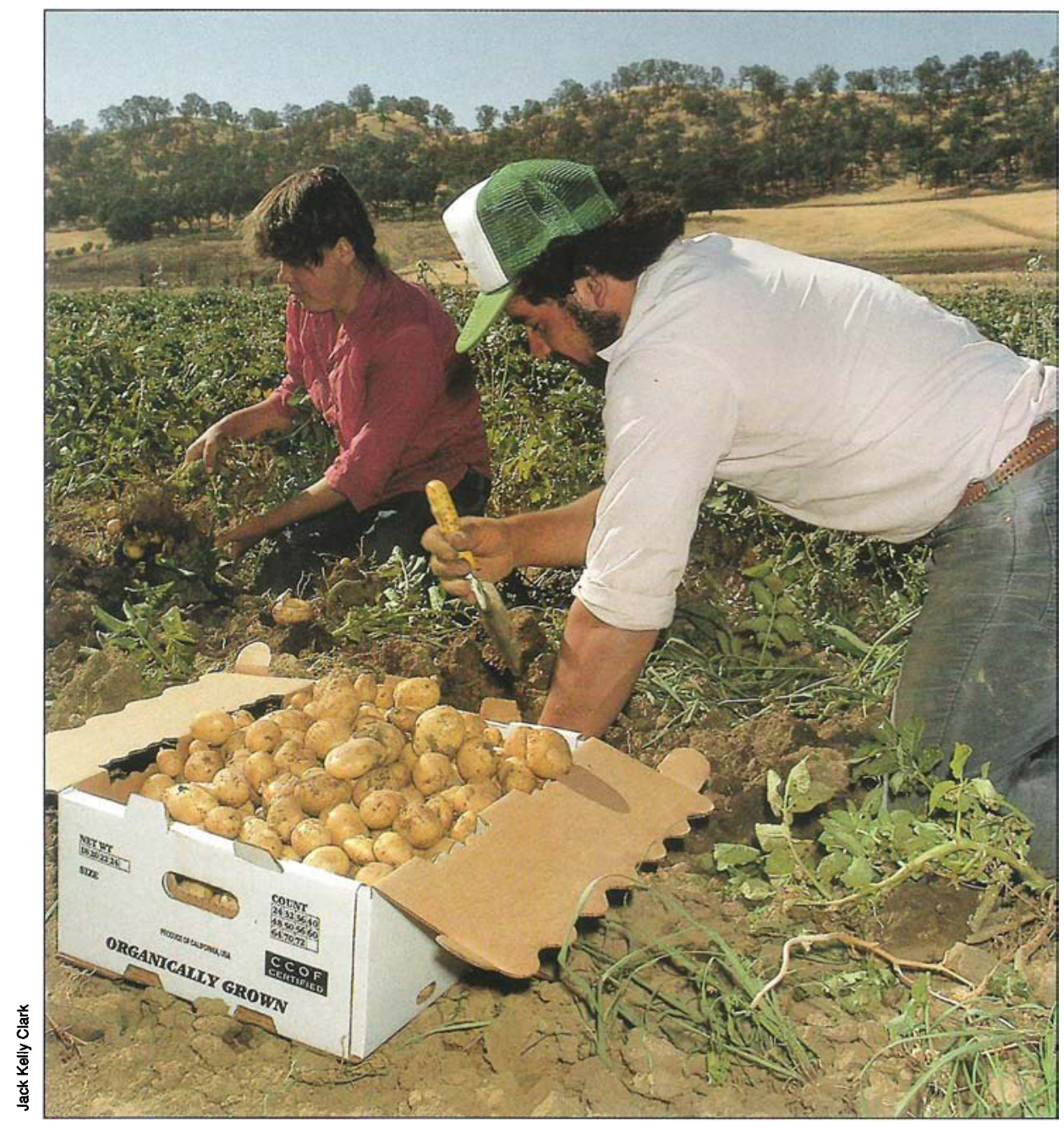

ing dramatically decrease the amount of residue that is ultimately consumed. However, processes such as food concentration or dehydration may increase levels of pesticide residues or pesticide breakdown products. Studies have shown, nonetheless, that concentrated and dehydrated food products typically contain residues below tolerance levels.

For example, the FDA annually conducts a Total Diet Study involving "market baskets" of selected food items, including meat and poultry. Four market baskets, each in a different region of the country, are filled with the same 234 food items. These foods are prepared in a

"ready-to-eat" fashion before they are analyzed for residues.

Although the Total Diet Study suffers from statistical limitations (due to small sample size) and does not detect residues of all possible pesticides, its annual findings have been consistent and are generally considered to be the most accurate representation of human exposure to pesticides in the United States.

Results from recent FDA Total Diet Studies indicate that human exposure to pesticides is typically far below the established acceptable daily intakes. Exposures normally range from $0.1 \%$ to $1 \%$ of the
ADIs, depending on the individual pesticide and the population subgroup for which consumption estimates are based. Assuming a safety factor of 100 which is commonly used when establishing ADIs, these ranges represent exposures that are 10,000 to 100,000 times lower than doses that have not caused any observed effects in long-term animal toxicology studies.

\section{Consumer alternatives}

Because of personal concern about pesticide residues in food, some consumers are taking action.

Individual actions can include washing produce with water (soap or detergent is not recommended) before eating it. The effectiveness of washing depends on the chemical and the commodity. One study showed as much as $97 \%$ or as little as $9 \%$ may be removed by water washing. Washing does not remove systemic pesticides. However, systemics are typically registered for use early in the growing season so that residual amounts at harvest are below tolerance.

Other consumer alternatives include backyard gardening without pesticides, and supporting alternative agricultural practices that eliminate or reduce the use of pesticides in growing crops.

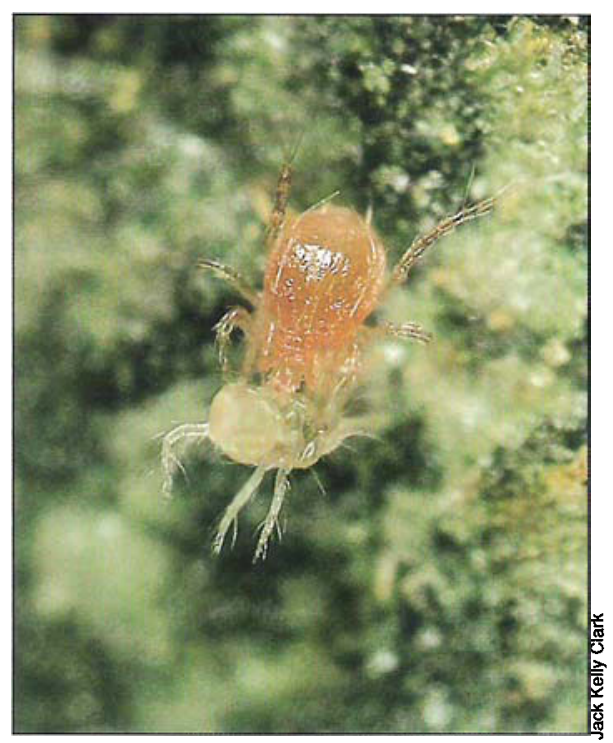

At left, farmworkers Lupe Garibay and Ricardo Fuentes hand harvest organically grown new potatoes at Capay fruit and vegetables, an organic farm in the Capay Valley, California.

Above, the predatory mite, Phytoseiulus persimilis, is shown here attacking a twospotted spider mite. This species is a very effective predator of spider mites when the weather is cool; releases have been used successfully to control mites in greenhouses and with some success in commercial strawberry fields.

Public actions include making preferences known to people who can make a difference, whether it is the local grocer or legislative representative.

People also can learn about and support programs which work to reduce pesticides in the environment. These include two programs in UC's Division of Agriculture and Natural Resources: Sustainable Agriculture Research and Education (916752-2379) and Integrated Pest Management (916-752-8350).

Despite the concerns surrounding the issue of pesticide residues in our food, there is general agreement that eliminating fresh fruits and vegetables from the diet is not a viable alternative. Eating a variety of foods, including two to four servings of fruit and three to five servings of vegetables a day, is still the cornerstone to a healthy diet.

G. A. Beall is Extension Communications Specialist, UC Davis; C. M. Bruhn is Extension Consumer Food Marketing Specialist, UC Davis; A. L. Craigmill is Extension Toxicologist, UC Davis; and Carl Winter was Extension Toxicologist, UC Riverside, and is now Extension Food Toxicologist, UC Davis. 